\title{
Tolerating Extreme Hyperkalemia in a Non-Compliant Dialysis Patient
} Attaway $\mathrm{A}^{* 1}$, Nanavati $\mathrm{A}^{2}$ and Dalal $\mathrm{S}^{3}$

${ }^{1}$ Pulmonary / Critical Care Fellow, University Hospital Case Western Reserve, Cleveland, Ohio, USA

${ }^{2}$ Internal Medicine Resident, Western Michigan University School of Medicine, Michigan, USA

${ }^{3}$ Nephrologist, Western Michigan Nephrology Center, Michigan, USA

${ }^{*}$ Corresponding author: Attaway A, Pulmonary / Critical Care Fellow, University Hospital Case Western

Reserve, Cleveland, Ohio, USA 44106, E-mail: amy.attaway@uhhospitals.org

Citation: Attaway A, Nanavati A, Dalal S (2015) Tolerating Extreme Hyperkalemia in a Non-Compliant

Dialysis Patient. J Case Rep Stud 3(5): 504. doi: 10.15744/2348-9820.3.504

Received Date: April 05, 2015 Accepted Date: October 10, 2015 Published Date: October 13, 2015

\begin{abstract}
Dialysis patients are known to tolerate high potassium due to the chronic hyperkalemia that is inherent in their disease. Despite this, most reports of extremely high potassium $(>9 \mathrm{mmol} / \mathrm{L})$ are in the setting of cardiac arrest. We describe the case of a 57 -year-old Caucasian male with past medical history significant for end stage renal disease known to be non-adherent to a low potassium diet as well as missed dialysis appointments. Stat potassium off of a basic metabolic panel was $10.2 \mathrm{mmol} / \mathrm{L}$, which was non-hemolyzed and confirmed on repeat testing. A stat EKG was performed which revealed peaked T waves, mildly increased PR interval (200 ms), and mild QRS widening $(142 \mathrm{~ms})$. There was no evidence of bradycardia or sinusoidal wave form. The patient received emergent dialysis and his EKG normalized.

Keywords: ESRD, Hyperkalemia
\end{abstract}

\section{Background}

End stage renal disease (ESRD) patients are known to tolerate hyperkalemia with less EKG changes as well as less life-threatening arrythmias leading to cardiac arrest. Mortality from hyperkalemia is estimated to be 3.1 per 1000 patient-years, whereas on a monthly basis $5-10 \%$ of hemodialysis patients have been observed to have hyperkalemia. $24 \%$ of ESRD patients require emergency dialysis for hyperkalemia at any given point $[1,2]$.

\section{Case Report}

Our patient is a 57-year-old Caucasian male with past medical history significant for end stage renal disease secondary to multiple myeloma and light chain deposition disease. He has a history of non-adherence to a low potassium diet and missed dialysis appointments. The patient had previously stated that he can "feel" when his potassium is too high, especially when he has lower extremity weakness, and he presents to the hospital when these symptoms are significant. Previous to this episode he has tolerated extremely high potassium up to $9.0 \mathrm{mmol} / \mathrm{L}$ with only mild EKG changes (Figure 1 ).

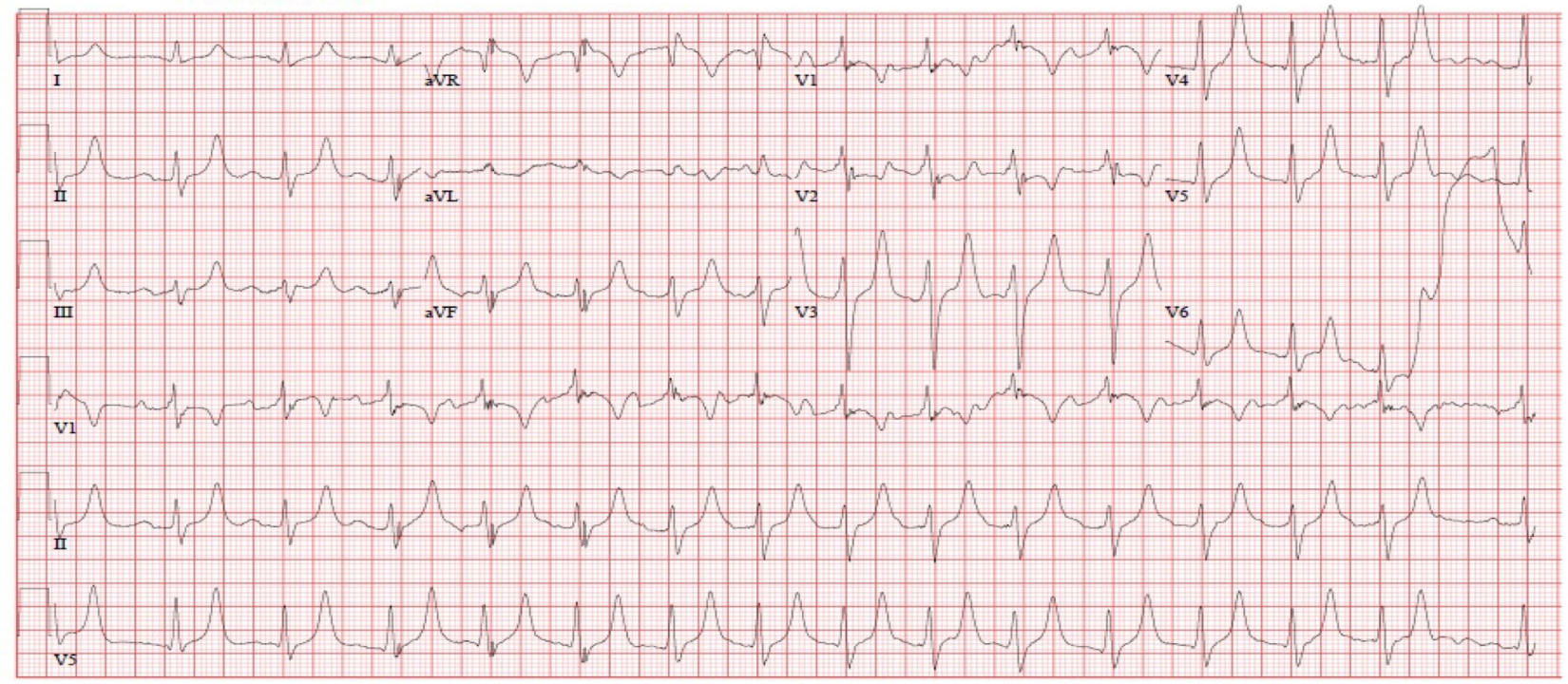

Figure 1: An EKG performed on the patient 1 week prior when his potassium was $9.2 \mathrm{mmol} / \mathrm{L}$. Here we see a ventricular rate of 95 , normal PR interval (150 ms), and narrow QRS (108 ms). There remained characteristic peaked T waves 
The patient presented in the early morning with lower extremity weakness. His last dialysis was three days prior. His blood pressure on admission was 210/116. He was somewhat confused. Exam revealed 4/5 strength in upper and lower extremities. Reflexes were hypoactive. Stat potassium off of a basic metabolic panel was $10.2 \mathrm{mmol} / \mathrm{L}$ which was non-hemolyzed and confirmed on repeat testing.

Chest X-ray was clear. EKG revealed peaked T waves, mildly increased PR interval (200 ms), and mild QRS widening (142 ms). There was no evidence of bradycardia or sinusoidal wave form. The patient received 10 units of insulin and 1 amp of D50 in the ER and went straight to the dialysis unit. He underwent emergent dialysis and both his mental status and EKG normalized (Figure 2) (Table 1).

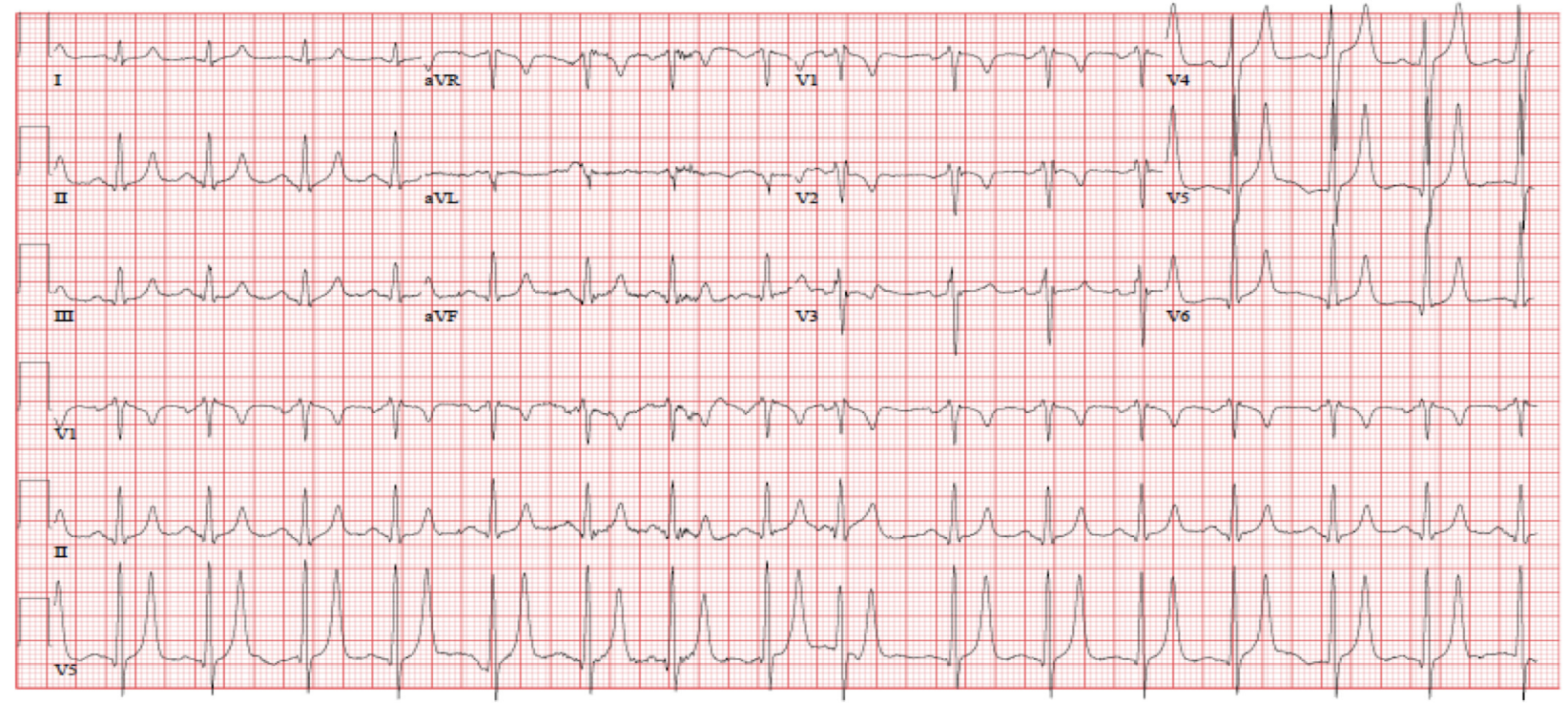

Figure 2: An EKG performed shortly after admission when the patient's potassium came back as $10.2 \mathrm{mmol} / \mathrm{L}$. He had sinus tachycardia (126) with characteristic peaked T waves, flattened $\mathrm{p}$ waves, an increased PR interval (200 ms), and QRS widening (142). While a sine wave is not yet present, there are early changes that could represent the beginnings of this morphology

\begin{tabular}{|c|c|c|}
\hline & Pre dialysis & Post dialysis \\
\hline Sodium $(\mathrm{mmol} / \mathrm{L})$ & 133 & 136 \\
\hline Potassium $(\mathrm{mmol} / \mathrm{L})$ & 10.2 & 7.3 \\
\hline Chloride $(\mathrm{mmol} / \mathrm{L})$ & 97 & 99 \\
\hline $\mathbf{C O}_{2}(\mathrm{mmol} / \mathrm{L})$ & 21 & 25 \\
\hline BUN $(\mathrm{mmol} / \mathrm{L})$ & 85 & 66 \\
\hline Creatinine $(\mathrm{mmol} / \mathrm{L})$ & 11.1 & 8.6 \\
\hline Calcium $(\mathrm{mmol} / \mathrm{L})$ & 9.9 & 9.3 \\
\hline Albumin $(\mathrm{g} / \mathrm{dL})$ & 3.1 & 3 \\
\hline
\end{tabular}

Table 1: Stat potassium was $10.2 \mathrm{mmol} / \mathrm{L}$ which was non-hemolyzed and confirmed on repeat testing

\section{Discussion}

Previous studies that have documented extreme hyperkalemia have all been reported in the setting of cardiac arrest, or else have not been as high as $10.2 \mathrm{mmol} / \mathrm{L}$ [3-5]. The only other case of extreme hyperkalemia without arrest was a patient reported in Hong Kong with a potassium of $10.7 \mathrm{mmol} / \mathrm{L}$. The 2007 case represented a 47 -year-old female who also had ESRD secondary to chronic glomerulonephritis on hemodialysis [6]. In our case, it was important to exclude pseudo-hyperkalemia, which can be the result of mechanical trauma during venipuncture or else secondary to thrombocytosis or severe leukocytosis. In our patient both his WBC and platelet counts were normal [7]. He also underwent venipuncture without a tourniquet, his sample was sent stat and reviewed as "non-hemolyzed" by the lab, and his repeat potassium level was the same value.

\section{Conclusion}

The phenomenon of chronic hyperkalemia in ESRD patients and physiologic tolerance of the cardiac myocardium has been well documented in previous studies. Patients with ESRD have been shown to tolerate hyperkalemia with less EKG changes [1,2]. There have been case reports of patients with severe hyperkalemia $(>9.0 \mathrm{~mm})$ that have had completely normal EKGs as well [7]. The ability to adapt the myocardium may be related to slow increases in potassium causing less electrophysiologic consequences. However, the exact mechanism remains unknown [8]. While hyperkalemia is a common problem for dialysis patients, those who tolerate extreme levels like our patient are the exception rather than the rule. Hyperkalemia remains a significant risk factor for mortality in the dialysis population and should be treated as a true medical emergency. 


\section{References}

1. Putcha N, Allon M (2007) Management of Hyperkalemia in Dialysis Patients. Semin Dial 20: 431-9.

2. Mattu A, Brady WJ, Robinson DA (2000) Electrocardiographic manifestations of hyperkalemia. Am J Emerg Med 18: $721-9$.

3. Tran HA (2005) Extreme Hyperkalemia. South Med J 98: 729-32.

4. Muck PM, Letterer S, Lindner U, Lehnert H, Haas CS (2012) Beating the odds -- surviving extreme hyperkalemia. Am J Emerg Med 30: $250 . e 1-4$.

5. Kes P, Orlić-Cunović D, Trubelja N (1995) A life-threatening complication of extreme hyperkalemia in a patient on maintenance hemodialysis. Acta Med Croatica 49: 147-50.

6. Lim YH, Hendricks J (2007) A case of nonfatal non-collapsed patient with extreme hyperkalemia. Hong Kong J Emerg Med 14: 228 -32.

7. Smellie WS (2007) Spurious hyperkalaemia. BMJ 334: 693-5.

8. Szerlip HM, Weiss J, Singer I (1986) Profound hyperkalemia without electrocardiographic manifestations. Am J Kidney Dis 7: 461-5.

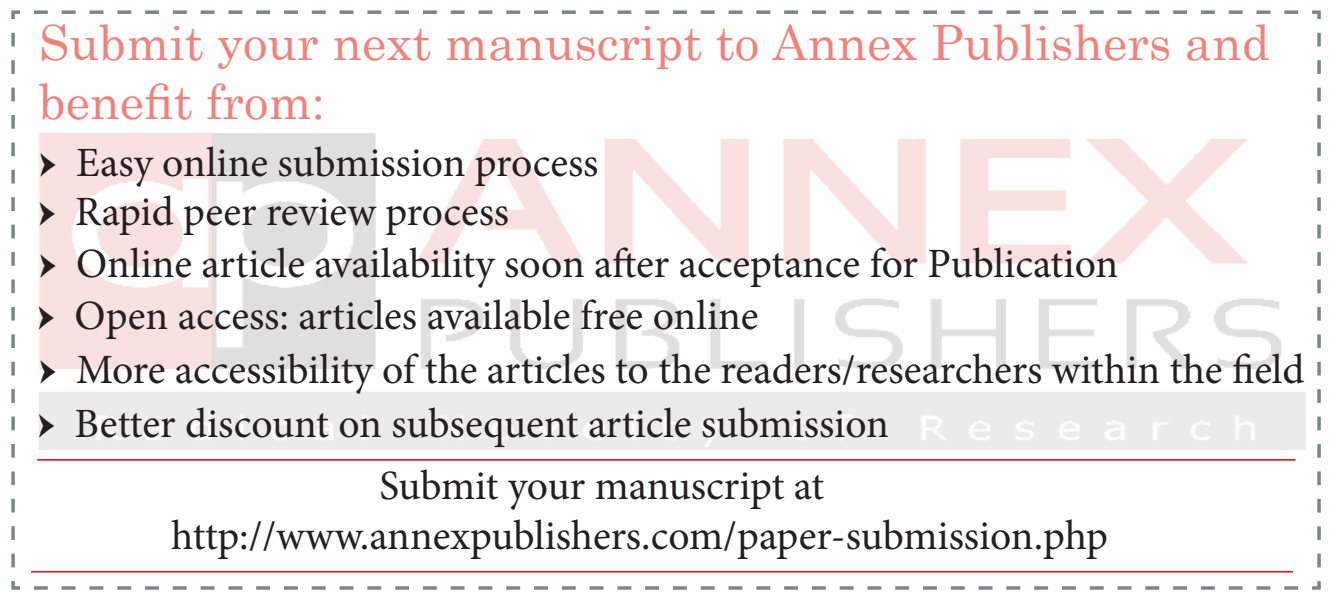

